\title{
Clinical outcomes of cardiac resynchronization therapy with and without a defibrillator in elderly patients with heart failure
}

\author{
Simon Christie BM ${ }^{1}$ (D) | Brett Hiebert MSc ${ }^{2}$ (D) | Colette M. Seifer MD (HONS), FRCP (UK) \\ Clarence Khoo MD, FRCPC ${ }^{3}$
}

${ }^{1}$ Max Rady College of Medicine, University of Manitoba, Winnipeg, Manitoba, Canada

${ }^{2}$ Cardiac Sciences Program, Winnipeg Regional Health Authority, Winnipeg,

Manitoba, Canada

${ }^{3}$ Section of Cardiology, University of Manitoba, Winnipeg, Manitoba, Canada

Correspondence

Clarence Khoo, Section of Cardiology, University of Manitoba, Winnipeg,

Manitoba, Canada.

Email: ckhoo@sbgh.mb.ca

\begin{abstract}
Background: Evidence regarding the incremental benefit of cardiac resynchronization therapy (CRT) with a defibrillator (CRT-D) versus without (CRT-P) in elderly patients with heart failure is limited. We compared mortality and cardiac hospitalisation between CRT-D and CRT-P in the elderly.

Methods: A retrospective chart review identified all consecutive patients with age $\geq 75$ with CRT implantation over the last 10 years at a Canadian tertiary care cardiac centre. Kaplan-Meier survival analyses and cumulative incidence curves were used to compare mortality and time to first cardiac hospitalisation, respectively, with CRT-D versus CRT-P over a 3 year period. Analyses were also repeated with propensity score matching based on age, sex, primary versus secondary prevention, date of implant, and Charlson Comorbidity Index.

Results: One hundred and seventy CRT patients were identified. A total of 128 received CRT-D while 42 received CRT-P. Median age was 79 (IQR 77-81), and the majority were male (83\%). CRT-P patients had a higher burden of comorbidities (Charlson score 7, IQR 6-8) than CRT-D patients (Charlson score 5, IQR 5-7; $P<0.001$ ). There was no significant difference in survival between the two groups in an unmatched comparison $(P=0.69)$ and with a propensity score-matched cohort $(P=0.91)$. Secondary prevention CRT-D patients had a higher risk of hospitalisation compared to primary prevention CRT-D patients; however, there was no significant difference in hospitalisation between the CRT-D and CRT-P groups.

Conclusion: This study suggests there is no significant difference in mortality or cardiac hospitalisation between CRT-D and CRT-P in elderly patients with heart failure.
\end{abstract}

KEYWORDS

cardiac resynchronization therapy, defibrillator, device, elderly, heart failure 


\section{1 | INTRODUCTION}

Cardiac resynchronization therapy (CRT) is a well-established treatment for individuals with medically optimised heart failure with New York Heart Association (NYHA) class II, III and ambulatory IV, a left ventricular ejection fraction (LVEF) of $\leq 35 \%$ and a prolonged QRS duration. ${ }^{1}$ Several studies have demonstrated the benefit of CRT on both mortality and morbidity. ${ }^{2-5}$

Given that there is overlap between patients qualifying for CRT as well as for an implantable cardioverter defibrillator (ICD) for primary prevention indications $s^{1,6,7}$, a majority of patients often receive CRT with an ICD (CRT-D) as opposed to CRT alone (CRT-P). Although the landmark COMPANION trial suggested no benefit of CRT-D over CRT-P ${ }^{2}$, subsequent analyses suggest an incremental benefit of CRT-D, especially in reducing arrhythmic death. ${ }^{8}$

While elderly patients were under-represented in early studies, recent literature suggests that older patients achieve a similar benefit from CRT compared to younger patients in regard to both mortality and morbidity when confounding medical issues are accounted for. ${ }^{9-11}$ However, there remains limited evidence regarding the incremental benefit of CRT-D versus CRT-P alone in this population, where increasing comorbidities and advanced age may reduce the relative risk of arrhythmic versus non-arrhythmic death. The aim of this study was to compare mortality and morbidity outcomes between elderly patients receiving CRT-P and CRT-D.

\section{2 | METHODS}

\section{1 | Patient population}

Consecutive patients receiving either a CRT-D or CRT-P at St. Boniface Hospital (Winnipeg, Canada) between 2007 and 2017 were identified through the pacemaker clinic database. St. Boniface Hospital is a tertiary care cardiac referral centre that is responsible for implantation and follow-up of all ICD and CRT devices in the province of Manitoba, with a catchment area that extends into North-Western Ontario and the territory of Nunavut. All patients over the age of 75 , either at their initial implant or replacement of an existing device, were included in this study. Patients with an ejection fraction (EF) $>35 \%$ at time of implant were excluded. Ethics approval was obtained from the St. Boniface Hospital Research Review Committee as well as from the University of Manitoba Bannatyne Campus Research Ethics Board.

\subsection{Selection of CRT-D candidates}

All referrals for CRT and ICD therapy were reviewed by a multidisciplinary team including electrophysiologists and pacemaker clinic nurses. For the majority of patients, the Canadian Cardiovascular Society 2005 ICD document was the most current guideline used to determine candidacy for CRT and ICD implantation. ${ }^{12}$ These guidelines were recently updated in 2016 , which impacted only a few study patients. Patients that qualified for a defibrillator were

\section{Impact statement}

We certify that this work is novel and adds to the literature evidence that adding a defibrillator to cardiac resynchronization therapy in individuals over 75 years old does not improve mortality.

\section{Brief summary}

Clinical outcomes of cardiac resynchronization therapy with and without a defibrillator in patients $\geq 75$ years old with medically optimised heart failure were compared. We found no significant difference in mortality or cardiac hospitalisations between the two groups, despite CRT-P patients having a higher comorbid index at baseline.

offered the option of either CRT-D or CRT-P, with the final determinant being strongly in favour of patient preference.

\section{3 | Baseline characteristics}

Demographics and clinical data were obtained via electronic records for all patients. The Charlson Comorbidity Score ${ }^{13}$ was used to provide an assessment of baseline health prior to implant.

\subsection{Assessment of left ventricular function and dimensions}

A combination of echocardiography, MUGA scans, MIBI scans, as well as left ventricular angiography were used to determine each patient's LVEF, with the most recent recording prior to implantation used in the analysis. With echocardiography-derived LVEF, a modified Simpson's biplane technique was used where possible. When only visual assessment was available, the lower number of the LVEF range was used for analysis. Timing and frequency of LVEF measurements were driven by routine clinical practise.

\section{5 | Outcomes \& follow-up}

Data for follow-up assessment were obtained from the pacemaker clinic database, the heart failure clinic database (where available), as well as electronic patient records.

Outcomes of interest included mortality and cardiac hospitalisation as tracked by the electronic patient records and databases. Cardiac hospitalisation included hospital admissions for any cardiac related event as well as unexplained syncope.

\section{6 | Statistical analysis}

Baseline patient characteristics were compared between the CRT-P and CRT-D cohorts. Categorical variables were compared using a 
chi-square or Fisher's exact test, continuous variables were compared using a Mann-Whitney test. Propensity scores were calculated for both CRT-D and CRT-P patient cohorts considering age, sex, Charlson comorbidity score, implantation indication (primary vs. secondary) and date of implant. A 1:1 propensity score match was performed using a Greedy Matching algorithm. ${ }^{14}$ All comparisons were two-tailed with a pre-defined $\alpha=0.05$.

Kaplan-Meier survival curves were developed for unmatched and matched cohorts to visualise survival rates between cohorts and were compared using a log-rank test. Cumulative incidence curves were developed for unmatched and matched cohorts to visualise the rates of heart failure hospitalisation between cohorts and compared using a Gray's test. Univariable and multivariable Cox proportional hazards regression analysis was undertaken on the unmatched cohort to identify the independent risk factors associated with survival time and time to heart failure hospitalisation for the entire cohort and the CRT-D and CRT-P cohorts separately. Death was considered a competing risk in the Cox proportional hazard models for the time to heart failure hospitalisation outcome. All final multivariable models were developed via a stepwise selection process. The selection required that a variable would have a score test $P$-value of $<0.05$ for entry into the model and a Wald test $P$-value of $<0.05$ to remain in the model. All analyses were performed using SAS version 9.3 (SAS Institute Inc., Cary, NC).

\section{3 | RESULTS}

\section{1 | Baseline characteristics}

Baseline characteristics of the study population are summarised in Table 1. A total of 170 patients with age $\geq 75$ years having a device-related procedure were included, of which the majority received their index device implant $(n=112)$, while the remainder received replacement of an existing device $(n=58)$. There were more CRT-D implanted $(n=128)$ versus CRT-P $(n=42)$. Within the CRT-D subgroup, the majority $(n=104)$ were implanted for primary prevention indications. Two patients in the CRT-P category had secondary prevention indications. One of these patients refused a defibrillator, while the other patient had an episode of bradycardiarelated Torsades de Pointes requiring pacing. 70 patients received follow-up EF assessments. Patients were followed for a median of 2.8 years.

The median age was 79 (IQR 77-81), with the minority (37\%) having severe LV dysfunction with an LVEF $<20 \%$. There were more males than females ( $n=141$ vs. 29), which was more pronounced in the CRT-D group than the CRT-P group ( $88 \%$ males vs. $67 \%$ males, respectively; $P<0.001$ ). Patients with ischaemic aetiology comprised a larger percentage of the CRT-D group than the CRT-P group ( $87 \%$ vs. $48 \%$, respectively; $P<0.001$ ). CRT-P patients tended to be older and have more comorbidities than the CRT-D patients (81 years old vs. 79 years old, respectively;
$P<0.001$, Charlson comorbidity score: 7 vs. 5 respectively; $P<0.001)$.

\section{2 | Differences in mortality between CRT-D and CRT-P}

The primary end point of mortality occurred in $47 / 128(36.7 \%)$ in the CRT-D group and 12 of $42(28.6 \%)$ in the CRT-P group. Kaplan-Meier analysis show no difference in survival curves between CRT-P and CRT-D implanted for both primary and secondary prevention indications over a 3-year follow-up (Figure 1A; $P=0.69$ ).

\section{3 | Differences in cardiac hospital admissions between CRT-D and CRT-P}

Survival analysis using a competing risks model suggested a significant difference in cardiac hospitalisation rates over 3 years of follow-up (Figure $2 A ; P=0.03$ ). By 2 years of follow-up, there was a marked increase in hospitalisation in the secondary prevention CRT-D group, whereas very little separation is seen between the primary prevention CRT-D and the CRT-P groups.

\section{4 | Propensity matched analysis of mortality and cardiac hospitalisation between CRT-D and CRT-P}

Propensity-score matching in a 1:1 fashion identified 27 CRT-D and 27 CRT-P patients matched for age, sex, Charlson comorbidity score, implantation indication (primary vs. secondary) and date of implant (Table 2). There was no difference in survival by Kaplan-Meier analysis over 3 years of follow-up between patients receiving CRT-D versus CRT-P (Figure $1 \mathrm{~B} ; \mathrm{P}=0.91$ ).

A competing risks model of cardiac admissions in this propensityscore matched group suggested a trend towards increased cardiac hospitalisation in CRT-D patients, with divergence of the curves by 1 year; however, this did not reach statistical significance (Figure 2B; $P=0.20)$.

\section{5 | Univariable and multivariable predictors of mortality}

Chronic kidney disease (CKD) was associated with increased mortality (HR 2.34, Cl 1.20-4.59, $P=0.013$ ), while ischaemic cardiomyopathy appeared to be protective (HR 0.48, Cl 0.24-0.95, $P=0.034)$. Following multivariable analysis, they remained independent predictors, with CKD associated with increased mortality (HR 3.00, Cl 1.44-6.25, $P=0.003$ ), and ischaemic cardiomyopathy associated with reduced mortality (HR 0.41, Cl 0.19-0.89, $P=0.024)$. Neither age, sex, Charlson score nor the decision to implant a CRT-D versus a CRT-P were independent predictors of mortality. 
TAB LE 1 Baseline characteristics of total study cohort

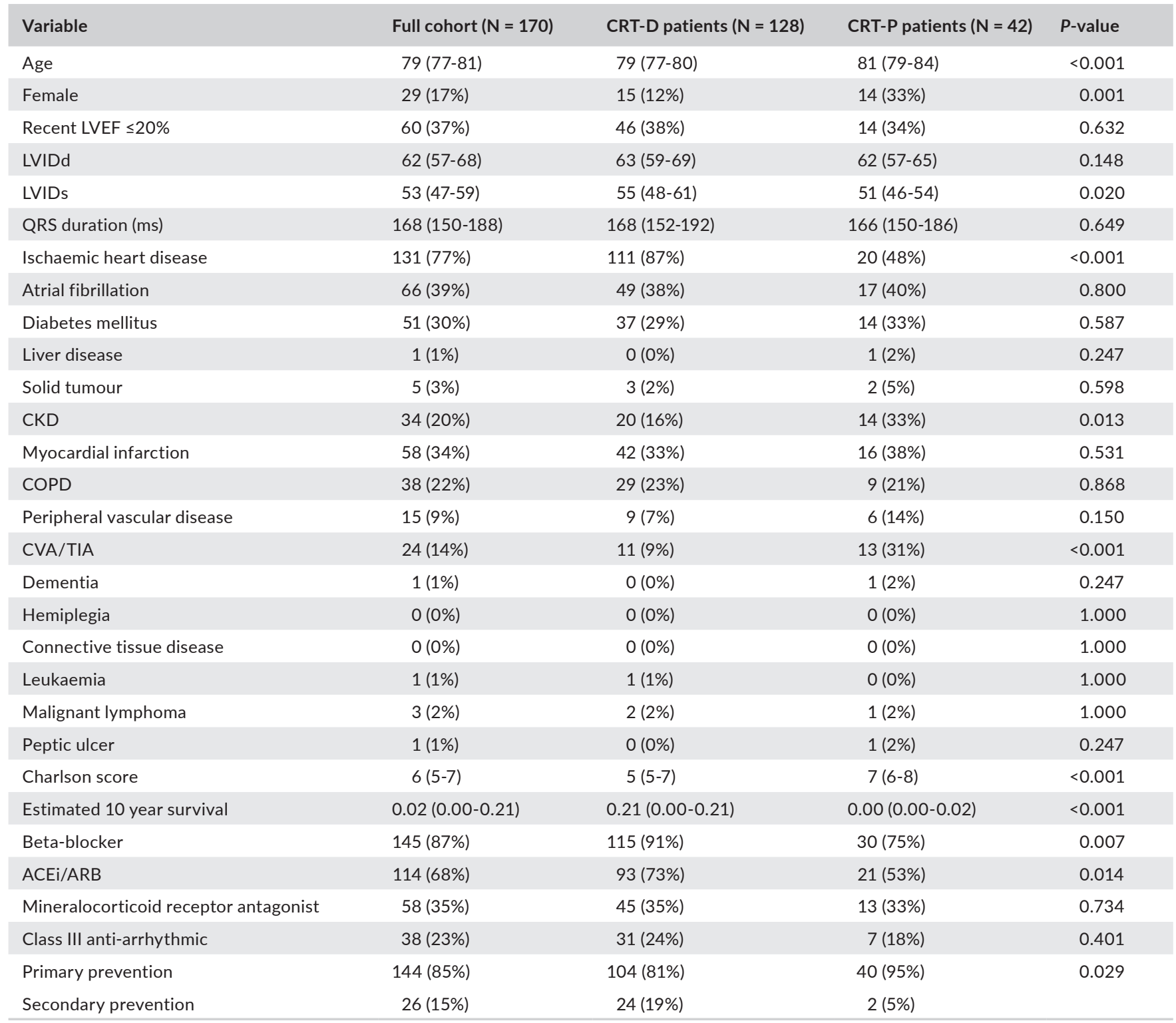

ACEi, angiotensin-converting-enzyme inhibitor; ARB, angiotensin II receptor blocker; LVIDd, LV end diastolic dimension (mm); LVIDs, LV end systolic dimension ( $\mathrm{mm}$ ).

Continuous variables expressed as median (quartile 1-quartile 3) compared using Mann-Whitney test, categorical variables expressed as $\mathrm{N}$ (\%) on nonmissing values compared using chi-square test or Fisher's exact test.

In the CRT-P sub-group, a secondary prevention indication was associated with a marked increase in mortality, but this did not remain significant after multivariable analysis.

\subsection{Univariable and multivariable predictors of cardiac hospitalisation}

Ischaemic cardiomyopathy ( $\mathrm{HR} 0.45, \mathrm{Cl} 0.22-0.92, P=0.029$ ), CKD (HR 3.34, Cl 1.68-6.62, $P<0.001$ ), as well as a secondary prevention indication (HR 2.28, Cl 1.09-4.77, $P=0.029$ ) were significant univariable predictors associated with cardiac hospitalisation. Following multivariable analysis, CKD (HR 3.55, Cl 1.72-7.35, $\mathrm{P}<0.001$ ) and a secondary prevention indication (HR $3.05, \mathrm{Cl}$
1.36-6.84, $P=0.007$ ) continued to remain independent predictors of increased hospitalisation. A more recent implant date was associated with increased hospitalisation (HR 1.28, Cl 1.05-1.57, $P=0.013)$.

\section{7 | ICD therapy}

Among patients in the CRT-D group, 17 in the primary prevention group and 10 in the secondary prevention group had at least one ventricular arrhythmia requiring device therapy, i.e. either shock or anti-tachycardia pacing (ATP). Appropriate ICD therapy was not a significant predictor of mortality in univariable analysis (HR $0.99 \mathrm{Cl}$ $0.40-2.42 P=0.973$ ). 

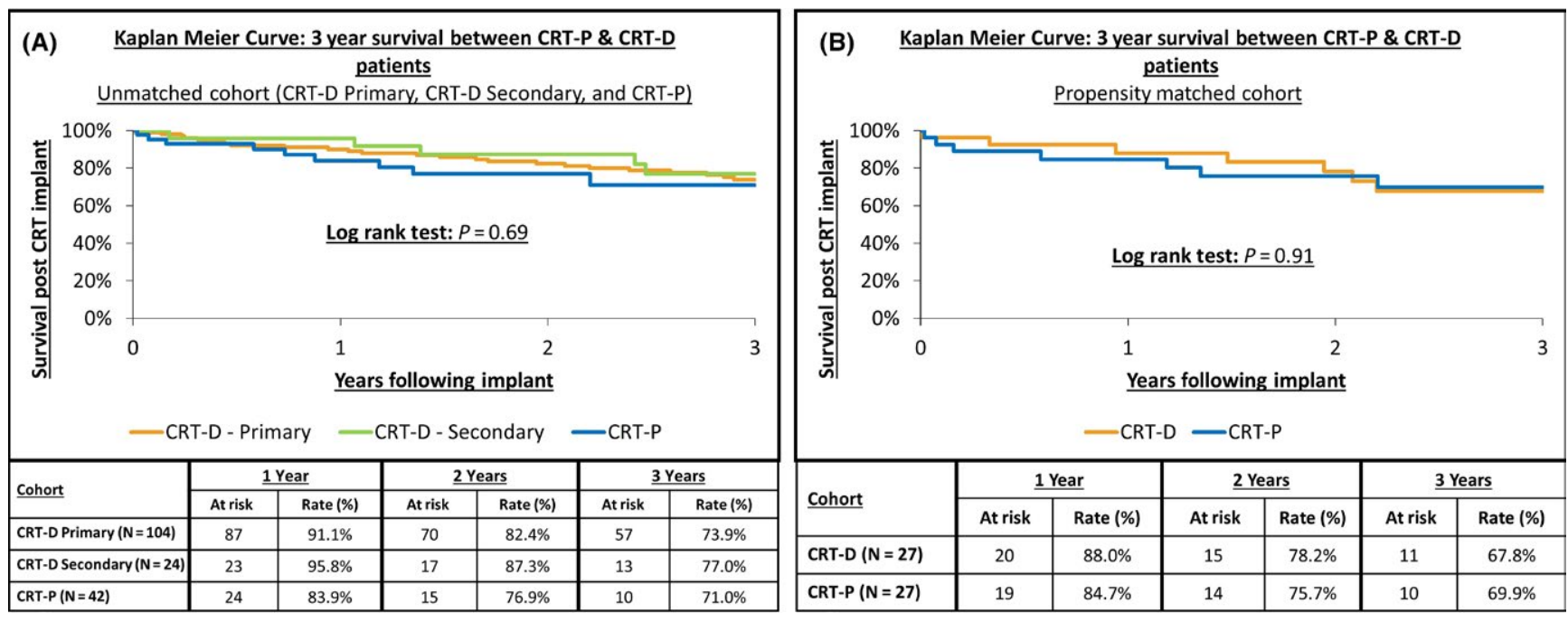

FIG URE 1 (A, B) 3-year survival between CRT-P \& CRT-D patients-unmatched and propensity matched cohorts
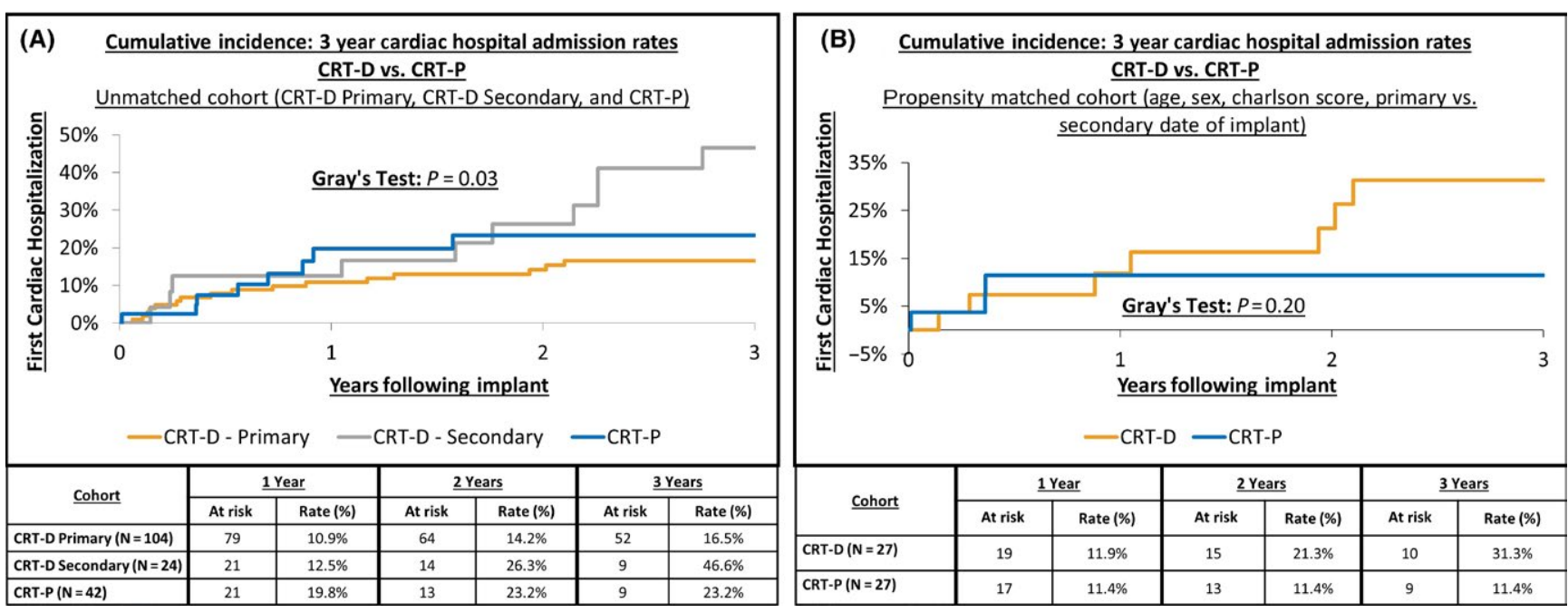

FIGURE 2 (A, B) 3 year cardiac hospital admission rates CRT-P versus CRT-D-unmatched and propensity matched cohorts

\section{4 | DISCUSSION}

Our study shows that in elderly patients with medically-optimised heart failure who qualify for CRT, there is no significant incremental mortality benefit of a CRT-D over a CRT-P. There was also no significant difference in cardiac hospitalisation between CRT-D and CRT-P patients, although patients with a secondary prevention indication were at increased risk.

These findings are in contrast to that of the COMPANION trial', which showed an incremental survival benefit of CRT-D over CRT-P, but no difference in the combined endpoint of mortality or hospitalisation. The population in the landmark study, however, was younger (median age $\sim 67$ years) with a recent diagnosis of CHF (median time from diagnosis $~ 3.6$ years). In comparison, our study investigated an older population of patients with a high burden of medical comorbidities where the potential incremental benefit of a CRT-D over a CRT-P may be attenuated.

The additional benefit of CRT-D over CRT-P is in the prevention of arrhythmic death. One explanation for the findings in this study is that the majority of elderly patients do not experience sudden cardiac death (SCD); rather, most deaths are due to worsening CHF or other comorbid illnesses. Accordingly, our results are in keeping with the findings of the CeRtiTuDe registry, which was a prospective, multicentre French registry of all CRT system implants. ${ }^{15}$ CRT-P patients were on average 10 years older than CRT-D patients, and were more likely to have other comorbid health issues, including renal insufficiency. CRT-P patients were found to have had a greater risk of mortality compared with CRT-D, but when cause-of-death analysis was performed it was found that the excess mortality among CRT-P patients was almost entirely related to non-SCD. 
TAB LE 2 Baseline characteristics of propensity-matched cohort

\begin{tabular}{|c|c|c|c|c|}
\hline Variable & Full cohort $(N=54)$ & CRT-D patients $(\mathrm{N}=27)$ & CRT-P patients $(\mathrm{N}=27)$ & $P$-value \\
\hline Female & $10(19 \%)$ & 5 (19\%) & 5 (19\%) & 1.000 \\
\hline QRS duration (ms) & $169(154-190)$ & 172 (154-190) & $166(158-196)$ & 0.931 \\
\hline Ischaemic heart disease & $33(61 \%)$ & $20(74 \%)$ & $13(48 \%)$ & 0.051 \\
\hline Diabetes mellitus & $18(33 \%)$ & $9(33 \%)$ & $9(33 \%)$ & 1.000 \\
\hline CKD & $14(26 \%)$ & $8(30 \%)$ & $6(22 \%)$ & 0.535 \\
\hline Myocardial infarction & $22(41 \%)$ & $12(44 \%)$ & $10(37 \%)$ & 0.580 \\
\hline COPD & $16(30 \%)$ & $11(41 \%)$ & 5 (19\%) & 0.074 \\
\hline Estimated 10 year survival & $0.02(0.00-0.21)$ & $0.02(0.00-0.21)$ & $0.02(0.00-0.21)$ & 0.747 \\
\hline Beta-blocker & 41 (80\%) & 23 (88\%) & $18(72 \%)$ & 0.173 \\
\hline ACEi/ARB & 32 (63\%) & $19(73 \%)$ & $13(52 \%)$ & 0.120 \\
\hline Mineralocorticoid receptor antagonist & $13(25 \%)$ & $6(23 \%)$ & $7(28 \%)$ & 0.687 \\
\hline Class III anti-arrhythmic & $7(14 \%)$ & $3(12 \%)$ & $4(16 \%)$ & 0.703 \\
\hline Primary prevention & $50(93 \%)$ & $25(93 \%)$ & $25(93 \%)$ & 1.000 \\
\hline Secondary prevention & $4(7 \%)$ & $2(7 \%)$ & $2(7 \%)$ & \\
\hline
\end{tabular}

ACEi, angiotensin-converting-enzyme inhibitor; ARB, angiotensin II receptor blocker.

Continuous variables expressed as median (quartile 1-quartile 3) compared using Mann-Whitney test, categorical variables expressed as $\mathrm{N}$ (\%) on nonmissing values compared using chi-square test or Fisher's exact test.

The benefit of an ICD in patients with a non-ischaemic cardiomyopathy has recently been brought into question by the DANISH trial, where there was no difference conferred by an ICD in the endpoints of all-cause mortality or SCD. ${ }^{16}$ The majority (58\%) of patients in the study received a CRT, with equal weighting of CRT-P and CRT-D. Moreover, subgroup analysis suggests that the lack of benefit of an ICD was largely driven by patients older than 68 years of age. This is again in agreement with the findings of our study.

Medical comorbidities strongly influence the risk of non-cardiac death, and may influence the decision to implant a CRT-P in clinical practise. The Charlson comorbidity score is a validated method of measuring the burden of comorbidities, and has been shown to strongly predict all-cause mortality. ${ }^{13}$ In our patient population, CRT-P patients had a significantly higher Charlson comorbidity score, with a predicted 10-year survival markedly lower than that for CRT-D patients. The lack of incremental benefit in survival with CRT-D is thus more striking given that the predicted survival was in favour of CRT-D based on comorbid burden alone. It is sobering to note that in our study, only $21 \%$ of CRT-D patients over the age of 75 were expected to be alive in 10 years. This again confirms that there is a strong competing risk for non-arrhythmic death in this population over arrhythmic SCD.

Of the comorbid conditions captured in this study, patients with CKD were at increased risk of mortality regardless of whether they receive CRT-D or CRT-P. This finding agrees with the existing literature that shows a higher risk of mortality in CRT patients with severe CKD. Patients with stage IV/V CKD have been estimated to have a 5 year mortality of up to $62.2 \%$ despite the implantation of a CRT-D. ${ }^{17}$ The literature supports an established benefit of CRT in the CKD population. The CARE-HF study found that patients with CKD had greater benefit from CRT over optimised medical therapy alone. ${ }^{3}$ There even appears to be a modest, but significant reduction in mortality and heart failure admission by the addition of a CRT-D over an ICD alone in patients with stage III/IV CKD. ${ }^{18}$ In contrast, the benefit of an ICD in this population is less well established. A recent meta-analysis showed that while there was a reduction in mortality with ICD in stage III CKD patients, there was no appreciable mortality benefit in patients with stage IV/V CKD. ${ }^{19}$ Given the poor overall prognosis of elderly patients with severe CKD and the uncertain benefit of an ICD in this population, it may be argued that a CRT-P to reduce CHF admissions would be a preferable treatment modality.

An ischaemic cardiomyopathy aetiology was associated with a decreased risk of both mortality and hospitalisation. The benefit of ICD or CRT implantation in ischaemic patients is well documented. In the MADIT-II study, patients implanted with a defibrillator had improved survival over those treated with conventional medical therapy alone. ${ }^{20}$ The subsequent MADIT-CRT study revealed that eligible patients with ischaemic cardiomyopathy had a reduction in hospitalisation with CRT-D rather than ICD alone. ${ }^{4}$ As noted above, 
this is in contrast with non-ischaemic aetiologies, where the benefit of an ICD has been recently thrown into question by the DANISH trial.

The 2005 Canadian Cardiovascular Society ICD guidelines were used clinically as selection criteria ${ }^{12}$, and differences in recommendations for non-ischaemic patients may have resulted in an enrichment of higher risk patients in this group by excluding lower risk individuals that would otherwise be implanted in the ischaemic group. Whereas indications for ICD implantation in ischaemic cardiomyopathy patients included those with LVEF $<35 \%$ with NYHA class I-III, the indications in non-ischaemic cardiomyopathy patients included only patients with LVEF $<30 \%$ with NYHA class II-III. As a result, lower risk non-ischaemic patients in the LVEF 30\%-35\% range, or with NYHA class I functional class were excluded, which may have thus increased the risk of events in the study group. In addition, a longer duration of optimal medical therapy for non-ischaemic patients-12 months versus 3 months-was recommended by the guidelines. This thereby also excluded nonischaemic patients with subsequent LVEF recovery and a favourable natural history.

In our study, secondary prevention patients were at greater risk of requiring hospitalisation for a cardiac cause. It is well documented in earlier studies that patients with secondary prevention indications are at greater risk of requiring an appropriate therapy from their ICD. ${ }^{21-23}$ In addition, the time from implantation to the first event occurs earlier in these patients. There is a well-known "shock-paradox" detailed in the ICD literature where patients that receive more appropriate shocks have an increased risk of mortality. ${ }^{24-27}$ There is still some debate as to whether the shocks themselves worsen heart failure or whether they are a marker of progressive disease.

SCD-HeFT demonstrated that patients who received at least one shock, whether appropriate or inappropriate, were at increased risk of mortality within the 4-year median follow-up period. The most common cause of death among those who received a shock was progressive heart failure. ${ }^{24}$ These results have been replicated in both the ALTITUDE survival study that showed that patients who received shock therapy were at increased risk of mortality compared to those who did not receive a shock ${ }^{25}$, and a large multi-centre study that showed that patients who received at least one shock had significantly increased risk of mortality compared to ATP or no ICD therapies. ${ }^{26}$ It is for these reasons that we decided to separate primary and secondary prevention patients during analysis.

Interestingly, despite the increased risk of appropriate therapy and hospitalisation among secondary prevention patients, we did not find any difference in mortality in comparison to primary prevention patients. It could be that the relatively short follow-up period of this study did not leave sufficient time for the differences in mortality to manifest. Another explanation for this finding is that the higher degree of comorbid illness and other competing causes of death seen in this older population results in a levelling of the relative risks of secondary versus primary prevention. While AVID ${ }^{28}$,
$\mathrm{CIDS}^{29}$ and $\mathrm{CASH}^{30}$ established the role of secondary prevention ICD's in preventing all-cause and arrhythmic death, a meta-analysis of the sub-group of patients $>75$ years in these trials showed no reduction in either all-cause or arrhythmic death. This was postulated to be driven by an increase in competing non-arrhythmic deaths in this population. ${ }^{31}$ While the role of an ICD in secondary prevention patients was not the focus of this study, this finding agrees with our other results suggesting that consideration of a patient's age and comorbidities should factor into decision-making surrounding device implantation.

Overall, our study suggests that in elderly patients with CHF due to systolic dysfunction, implantation of a CRT-P may be associated with an equivalent risk of all-cause mortality and a reduction in cardiac hospitalisation compared with a CRT-D. As with all patients referred for device therapy, this finding reinforces the need for health care providers to carefully consider not just the LVEF, but also a patient's age, comorbidities and preferences when selecting which device would best suit the patient's needs. Clinicians may therefore consider using our findings to support the use of CRT-P in elderly patients with a high comorbid burden or other potential competing causes of non-arrhythmic death.

\section{1 | Limitations}

The main limitations of this study are the relatively small sample size as well as the 3-year duration of follow-up. One of the reasons for the relatively short follow-up period is that very few patients were being implanted with CRT-P prior to 2011. Although there has been a steady increase in the number of CRT-P implantations over the last several years, there remains a limited amount of follow-up data on many of our CRT-P patients.

This study was also limited due to its retrospective design. Incomplete datasets, such as follow-up EF assessment, limited the ability to utilise certain variables in our analysis.

\section{5 | CONCLUSIONS}

This study suggests there is no significant difference in mortality between CRT-D and CRT-P in patients over the age of 75 with heart failure receiving optimal medical therapy implanted for primary prevention indications. Among CRT-D patients, secondary prevention confers a higher risk for hospitalisation than primary prevention. Individuals with CKD are at increased risk of mortality in both CRT-D and CRT-P populations. In elderly patients with significant comorbidities, CRT-P may thus be a reasonable option over CRT-D. Further investigation into the differences between CRT-D and CRT-P in the elderly is warranted.

\section{ACKNOWLEDGEMENTS}

This research did not receive any specific grant from funding agencies in the public, commercial or not-for-profit sectors. 


\section{DECLARATIONS OF INTEREST}

None.

\section{AUTHOR CONTRIBUTIONS}

Acquisition of data was done by Simon Christie and Clarence Khoo. Statistical analysis was performed by Brett Hiebert. All authors contributed to the text of the manuscript.

\section{ORCID}

Simon Christie iD http`s://orcid.org/0000-0003-4910-6693

Brett Hiebert iD https://orcid.org/0000-0003-4701-7144

Colette M. Seifer (iD https://orcid.org/0000-0001-9841-7960

Clarence Khoo (iD https://orcid.org/0000-0002-3979-9871

\section{REFERENCES}

1. Exner DV, Birnie DH, Moe G, et al. Canadian cardiovascular society guidelines on the use of cardiac resynchronization therapy: evidence and patient selection. Can J Cardiol. 2013;29(2):182-95. https://doi.org/10.1016/j.cjca.2012.10.006.

2. Bristow MR, Saxon LA, Boehmer J, et al. Cardiac-resynchronization therapy with or without an implantable defibrillator in advanced chronic heart failure. N Engl J Med. 2004;350(21):2140-50.

3. Cleland JGF, Daubert J-C, Erdmann E, et al. The effect of cardiac resynchronization on morbidity and mortality in heart failure. N Engl J Med. 2005;352(15):1539-49.

4. Moss AJ, Hall WJ, Cannom DS, et al. Cardiac-resynchronization therapy for the prevention of heart-failure events. N Engl J Med. 2009;361(14):1329-38.

5. Tang ASL, Wells GA, Talajic M, et al. Cardiac-resynchronization therapy for mild-to-moderate heart failure. N Engl J Med. 2010;363(25):2385-95.

6. Bennett M, Parkash R, et al. Canadian Cardiovascular Society/ Canadian Heart Rhythm Society 2016 implantable cardioverterdefibrillator guidelines. Can J Cardiol. 2017;33:174-88. https://doi. org/10.1016/j.cjca.2016.09.009.

7. Yancy CW, Jessup M, Bozkurt B, et al. 2013 ACCF/AHA guideline for the management of heart failure: a report of the American College of Cardiology Foundation/American Heart Association Task Force on Practice Guidelines. J Am Coll Cardiol. 2013;62(16):147-239. https://doi.org/10.1016/j.jacc.2013.05.019.

8. Barra S, Providencia R, Tang A, Heck P, Virdee M, Agarwal S. Importance of implantable cardioverter-defibrillator back-up in cardiac resynchronization therapy recipients: a systematic review and meta-analysis. J Am Heart Assoc. 2015;4(11):e002539. https://doi. org/10.1161/JAHA.115.002539.

9. Penn J, Goldenberg I, Moss AJ, et al. Improved outcome with preventive cardiac resynchronization therapy in the elderly: A MADITCRT substudy. J Cardiovasc Electrophysiol. 2011;22(8):892-7. https://doi.org/10.1111/j.1540-8167.2011.02011.x.

10. Verbrugge FH, Dupont M, De Vusser P, et al. Response to cardiac resynchronization therapy in elderly patients ( $\geq 70$ years) and octogenarians. Eur J Heart Fail. 2013;15:203-10. https://doi.org/10.1093/ eurjhf/hfs151.

11. Killu AM, Wu J-H, Friedman PA, et al. Outcomes of cardiac resynchronization therapy in the elderly. PACE. 2013;36:664-72. https:// doi.org/10.1111/pace.12048.
12. Tang AS, Ross H, Simpson CS, et al. Canadian Cardiovascular Society/ Canadian Heart Society position paper on implantable cardioverter defibrillator use in Canada. Can J Cardiol. 2005;21(Suppl A):11A-8A.

13. Charlson ME, Pompei $P$, Ales KL, MacKenzie CR. A new method of classifying prognostic comorbidity in longitudinal studies: development and validation. J Chronic Dis. 1987;40(5):373-83.

14. Rosenbaum PR. Observational Studies, 2nd ed. New York, NY: Springer Verlag;2002. https://doi.org/10.1007/978-1-4757-3692-2.

15. Marijon E, Leclercq C, Narayanan K, et al. Causes-of-death analysis of patients with cardiac resynchronization therapy: an analysis of the CeRtiTuDe cohort study. Eur Heart J. 2015;36:2767-76. https://doi.org/10.1093/eurheartj/ehv455.

16. Køber L, Thune JJ, Nielsen JC, et al. Defibrillator implantation in patients with nonischemic systolic heart failure. N Engl J Med. 2016;375(13):1221-30. https://doi.org/10.1056/NEJMoa16080 29.

17. Daly DD Jr, Maran A, Hyer JM, et al. The effect of chronic kidney disease on mortality with cardiac resynchronization therapy. PACE. 2017;39(8):863-9. https://doi.org/10.1111/pace.12883. The.

18. Friedman DJ, Singh JP, Curtis JP, et al. Comparative effectiveness of CRT-D versus de fi brillator alone in HF patients with moderate-tosevere chronic kidney disease. J Am Coll Cardiol. 2015;66(23):261829. https://doi.org/10.1016/j.jacc.2015.09.097.

19. Fu L, Zhou Q, Zhu W, et al. Do implantable cardioverter defibrillators reduce mortality in patients with chronic kidney disease at al stages? Int Heart J. 2017;58(3):371-7.

20. Huang DT, Sesselberg HW, Nitt SMC, et al. Improved survival associated with prophylactic implantable defibrillators in elderly patients with prior myocardial infarction and depressed ventricular function: a MADIT-II substudy. J Cardiovasc Electrophysiol. 2007;18(8):8338. https://doi.org/10.1111/j.1540-8167.2007.00857.x.

21. Stockburger M, Krebs A, Nitardy A, Knaus T, Dietz R. Survival and appropriate device interventions in recipients of cardioverter defibrillators implanted for the primary versus secondary prevention of sudden cardiac death. PACE. 2009;32(Suppl. 1):S16-20.

22. Sweeney MO, Wathen MS, Volosin K, et al. Appropriate and inappropriate ventricular therapies, quality of life, and mortality among primary and secondary prevention implantable cardioverter defibrillator patients. Circulation. 2005;111(22):2898-906. https:// doi.org/10.1161/CIRCULATIONAHA.104.526673.

23. Wilkoff BL, Hess M, Young J, William T. Differences in tachyarrhythmia detection and implantable cardioverter defibrillator therapy by primary or secondary prevention indication in cardiac resynchronization therapy patients. J Cardiovasc Electrophysiol. 2004;15(9):1002-9. https://doi.org/10.1046/j.1540-8167.2004. 03625.x.

24. Bardy GH, Lee KL, Mark DB, et al. Amiodarone or an implantable cardioverter-defibrillator for congestive heart failure. N Engl J Med. 2005:352(3):225-37.

25. Saxon LA, Hayes DL, Gilliam FR, et al. Long-term outcome after ICD and CRT implantation and influence of remote device follow-up The ALTITUDE Survival Study. Circulation. 2010;122(23): 2359-67. https://doi.org/10.1161/CIRCULATIONAHA.110.960 633.

26. Strickberger SA, Canby R, Cooper J, et al. Association of antitachycardia pacing or shocks with survival in 69,000 patients with an implantable defibrillator. J Cardiovasc Electrophysiol. 2017;28(4):416-22. https://doi.org/10.1111/jce.13170.

27. Poole JE, Johnson GW, Hellkamp AS, et al. Prognostic importance of defibrillator shocks in patients with heart failure. N Engl J Med. 2008;359:1009-17.

28. Zipes DP, Wyse DG, Friedman PL, et al. A comparison of antiarrhythmic-drug therapy with implantable defibrillators in 
patients resuscitated from near-fatal ventricular arrhythmias. N Engl J Med. 1997;337:1576-84.

29. Connolly SJ, Gent M, Roberts RS, et al. Canadian Implantable Defibrillator Study (CIDS): a randomized trial of the implantable cardioverter defibrillator against amiodarone. Circulation. 2000;101(11):1297-303.

30. Kuck K-H, Cappato R, Siebels J, Rüppel R. Randomized comparison of antiarrhythmic drug therapy with implantable defibrillators in patients resuscitated from cardiac arrest: The Cardiac Arrest Study Hamburg (CASH). Circulation. 2000;102(7):748-55.

31. Healey JS, Hallstrom AP, Kuck K, et al. Role of the implantable defibrillator among elderly patients with a history of life-threatening ventricular arrhythmias. Eur Heart J. 2007;28:1746-9. https://doi. org/10.1093/eurheartj/ehl438.

How to cite this article: Christie S, Hiebert B, Seifer CM, Khoo C. Clinical outcomes of cardiac resynchronization therapy with and without a defibrillator in elderly patients with heart failure.

J Arrhythmia. 2019;35:61-69. https://

doi.org/10.1002/joa3.12131 